\title{
Human Hematopoietic Stem Cells Can Survive In Vitro for Several Months
}

\author{
Taro Ishigaki, ${ }^{1,2}$ Kazuhiro Sudo, ${ }^{1}$ Takashi Hiroyama, ${ }^{1}$ Kenichi Miharada, ${ }^{1}$ \\ Haruhiko Ninomiya, ${ }^{2}$ Shigeru Chiba, ${ }^{2}$ Toshiro Nagasawa, ${ }^{2}$ and Yukio Nakamura ${ }^{1}$ \\ ${ }^{1}$ Cell Engineering Division, RIKEN BioResource Center, Tsukuba, Ibaraki 305-0074, Japan \\ ${ }^{2}$ Division of Hematology, Institute of Clinical Medicine, University of Tsukuba, Tsukuba, Ibaraki 305-8575, Japan
}

Correspondence should be addressed to Yukio Nakamura, yukionak@brc.riken.jp

Received 22 September 2008; Revised 8 December 2008; Accepted 15 December 2008

Recommended by N. Chao

We previously reported that long-lasting in vitro hematopoiesis could be achieved using the cells differentiated from primate embryonic stem (ES) cells. Thus, we speculated that hematopoietic stem cells differentiated from ES cells could sustain long-lasting in vitro hematopoiesis. To test this hypothesis, we investigated whether human hematopoietic stem cells could similarly sustain long-lasting in vitro hematopoiesis in the same culture system. Although the results varied between experiments, presumably due to differences in the quality of each hematopoietic stem cell sample, long-lasting in vitro hematopoiesis was observed to last up to nine months. Furthermore, an in vivo analysis in which cultured cells were transplanted into immunodeficient mice indicated that even after several months of culture, hematopoietic stem cells were still present in the cultured cells. To the best of our knowledge, this is the first report to show that human hematopoietic stem cells can survive in vitro for several months.

Copyright (c) 2009 Taro Ishigaki et al. This is an open access article distributed under the Creative Commons Attribution License, which permits unrestricted use, distribution, and reproduction in any medium, provided the original work is properly cited.

\section{Introduction}

Identification of in vitro culture protocols that enable somatic stem cells to survive and proliferate will be of value not only for basic research but also clinical applications that require somatic stem cells. The development of an efficient method for in vitro proliferation of mesenchymal stem cells, for example, has allowed cultured mesenchymal stem cells to be used in clinical applications [1].

Although hematopoietic stem cells have been extensively analyzed and characterized [2], in vitro proliferation of these cells remains problematic using established culture methods $[3,4]$. In addition, the length of time that hematopoietic stem cells can survive in an in vitro culture system remains uncertain. CD34-positive $\left(\mathrm{CD} 34^{+}\right)$cells have been identified in long-term in vitro cultures of hematopoietic stem cells [59]. However, as none of these previous studies performed an in vivo assay of the cultured cells, such as transplantation into mice, it is uncertain whether hematopoietic stem cells with the capacity to reconstitute long-term in vivo hematopoiesis were present in these prolonged in vitro cultures.
We previously described a culture method that produced long-lasting in vitro hematopoiesis using non-human primate embryonic stem (ES) cells [10]. We speculated that hematopoietic stem cells derived from ES cells could sustain long-lasting in vitro hematopoiesis. To test this hypothesis, we initiated long-term in vitro cultures of human hematopoietic stem cells using the same culture method as previously [10]. In addition, we evaluated the in vivo function of cells cultured in vitro for several months by transplanting them into immunodeficient mice.

\section{Materials and Methods}

2.1. Cell Culture. We purchased human umbilical cord blood samples from the Cell Engineering Division of RIKEN BioResource Center (Tsukuba, Ibaraki, Japan). The ethical committee of the RIKEN Tsukuba Institute approved the use of human umbilical cord blood before the study was initiated. CD $34^{+}$hematopoietic stem/progenitor cells were collected from human umbilical cord blood using a magnetic cell sorting system, MACS CD34 Isolation kit (Miltenyi 
Biotec Inc., Sunnyvale, Calif, USA), according to the manufacturer's instructions.

Mouse-derived cell lines (OP9 and C3H10T1/2) were purchased from the Cell Engineering Division of RIKEN BioResource Center (Tsukuba, Ibaraki, Japan) and were cultured as follows: OP9 in Minimum Essential Medium$\alpha$ (MEM- $\alpha$; Invitrogen, Carlsbad, Calif, USA) containing $20 \%$ fetal bovine serum (FBS; Invitrogen, Calif, USA); C3H10T1/2 in Basal Medium Eagle (BME; Invitrogen) containing 10\% FBS (BioWest, Miami, Fla, USA). The cell lines were $\gamma$-irradiated ( $50 \mathrm{~Gy}$ ) before use as feeder cells.

$\mathrm{CD} 4^{+}$cells were cultured on feeder cells in a $100 \mathrm{~mm}$ dish in Iscove's modified Dulbecco's medium (IMDM; SIGMA, St Louis, Mo, USA) containing 10\% FBS (BioWest), $10 \mu \mathrm{g} / \mathrm{mL}$ bovine insulin, $5.5 \mu \mathrm{g} / \mathrm{mL}$ human transferrin, $5 \mathrm{ng} / \mathrm{mL}$ sodium selenite (ITS liquid MEDIA supplement; SIGMA-Aldrich, Mass, USA), 100 unit/mL penicillin, $100 \mu \mathrm{g} / \mathrm{mL}$ streptomycin, $2 \mathrm{mM}$ L-glutamine (PSQ; Invitrogen), $50 \mathrm{ng} / \mathrm{mL}$ stem cell factor (SCF; R\&D Systems, Minneapolis, Minn, USA), $50 \mathrm{ng} / \mathrm{mL}$ Flt-3 ligand (Flt3L; R\&D Systems), and $50 \mathrm{ng} / \mathrm{mL}$ thrombopoietin (TPO; R\&D Systems). The initial number of $\mathrm{CD} 34^{+}$cells placed in culture varied between experiments: $5 \times 10^{3}$ cells in Exp-OP9-A, Exp-10T1/2-A, and Exp-OP9-B; $4 \times 10^{4}$ cells in Exp-OP9-F and Exp-10T1/2-F; $5 \times 10^{4}$ cells in ExpOP9-C, Exp-OP9-E, Exp-10T1/2-E, Exp-OP9-H, and Exp$10 \mathrm{~T} 1 / 2-\mathrm{H} ; 8 \times 10^{4}$ cells in Exp-OP9-G and Exp-10T1/2G; $1 \times 10^{5}$ cells in Exp-10T1/2-I; $2 \times 10^{5}$ cells in ExpOP9-D and Exp-10T1/2-D. The letters "A" to "I" after Exp-OP9 or Exp-10T1/2 indicate 9 different umbilical cord blood samples derived from 9 different neonates. Samples A, B, C, F, G, H, and I were frozen after collection, while samples D and E were used immediately as fresh samples.

Twenty-four hours after initiation of culture, the medium together with any detached cells was removed and fresh medium was added to the culture. Thereafter, the medium was changed every 3-4 days (twice a week). The number of cells attached to the feeder cells increased gradually. Approximately four weeks after initiation of culture, attached cells were dissociated using a 0.25\% trypsin EDTA solution (SIGMA-Aldrich) and cultured again on new feeder cells. Thereafter, similar passages of attached cells were performed every 3-4 weeks.

The number of viable cells was assessed using an automated cell counter and an assay based on the trypan blue dye exclusion method, ViCell (BECKMAN COULTER, Fullerton, Calif, USA). The morphology of the cells was analyzed by microscopic examination after Wright staining (Muto Pure Chemicals, Tokyo, Japan).

2.2. Flow Cytometry. Cells were stained with monoclonal antibodies (MoAbs) and analyzed using a FACS Calibur (BD Biosciences, San Jose, Calif, USA). The following MoAbs were purchased from BD Biosciences: fluorescein isothiocyanate- (FITC-) conjugated MoAb against human CD14 (FITC-CD14), FITC-CD34, FITC-CD41a, and FITC-CD45; phycoerythrin-conjugated MoAb against human CD4(PE-CD4), PE-CD11b, PE-CD13, PE-CD34,
PE-CD45, PE-CD56, and PE-CD235a (Glycophorin A); allophycocyanin-conjugated MoAb against human CD3 (APC-CD3), APC-CD8, APC-CD19, APC-CD33, and APCCD45. PE-CD33 was purchased from eBiosciences (San Diego, Calif, USA). FITC-mouse IgG1, PE-mouse IgG1, APC-mouse IgG1, FITC-mouse IgG2a, and PE-mouse IgG2b were also purchased from $\mathrm{BD}$ Biosciences and were used as isotype controls. Cell viability was monitored by staining with propidium iodide (SIGMA-Aldrich). Flow cytometry data were analyzed using CellQuest (BD Biosciences) analysis software.

2.3. Colony Formation Assay. Cells $\left(1 \times 10^{4}\right)$ were cultured in a $35 \mathrm{~mm}$ dish with $1 \mathrm{~mL}$ of Methocult (H4435; Stem cell technology, Vancouver, British Columbia, Canada) for 10-14 days, and the number of separate colonies was determined by macroscopic morphology. Representative colonies were picked up and cell morphology was analyzed by microscopic examination after Wright staining (Muto Pure Chemicals).

2.4. Cell Transplantation into Mice. Eight-week-old male NOD/Shi-scid IL-2R $\gamma^{\text {null }}$ (NOG) mice were purchased from the Central Institute for Experimental Animals (Kawasaki, Kanagawa, Japan) and used within two weeks of delivery in all experiments. Prior to cell transplantation, the mice were given a sublethal dose of $\gamma$-rays (3.0 Gy). A $200 \mu \mathrm{L}$ cell suspension in phosphate-buffered saline (PBS; SIGMA) containing 5\% FBS (BioWest) was injected intravenously into the tail vein of each mouse. All experimental procedures on the mice were approved by the Institutional Animal Care and Use Committee of the RIKEN Tsukuba Institute.

\section{Results and Discussion}

3.1. Long-Lasting In Vitro Hematopoiesis from Human Hematopoietic Stem Cells. Human $\mathrm{CD}_{3}{ }^{+}$cells were cultured on feeder cells in the presence of SCF, Flt-3L, and TPO. We used the mouse-derived cell lines, OP9 and C3H10T1/2, as feeder cells. Both OP9 [10-15] and C3H10T1/2 [10, 1618] have been used in many studies to maintain in vitro hematopoiesis. As we show below, both OP9 and C3H10T1/2 cells supported long-lasting in vitro hematopoiesis from human hematopoietic stem cells.

About one week after initiation of culture, cobblestone areas were observed on the feeder cells (Figures 1(a) and $1(\mathrm{~b})$ ), indicating that human hematopoietic cells were proliferating. The numbers of cells attached to the feeder cells increased gradually (Figures $1(\mathrm{c})$ and $1(\mathrm{~d})$ ) and the numbers of detached cells, derived from the attached cells, also increased gradually. When the medium was changed, the numbers of detached cells in the medium were counted and the cells were subjected to analyses such as flow cytometric analysis. Detached cells were removed during the medium changes and were not cultured further in any of the experiments.

Detached cells were continuously produced for several months in experiments Exp-OP9-A, Exp-OP9-F, Exp-OP9H, Exp-10T1/2-A, and Exp-10T1/2-H (Figure 2). As we 


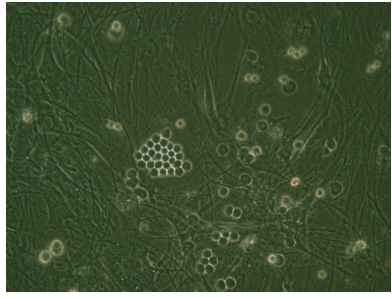

(a)

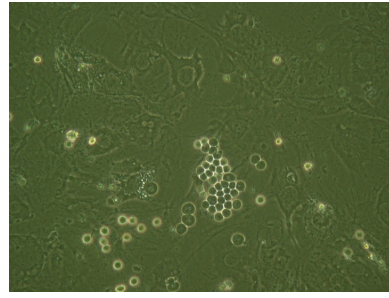

(b)

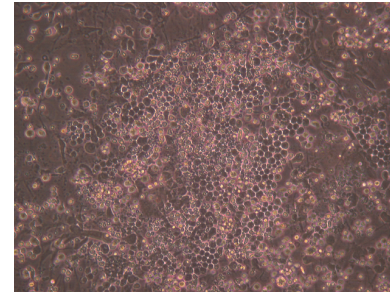

(c)

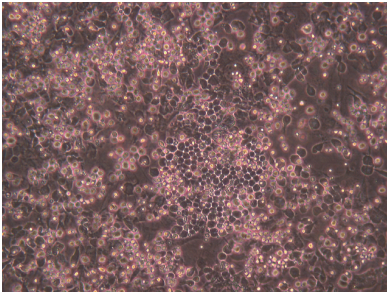

(d)

FIGURE 1: Appearance of the cells attached to feeder cells. Representative examples of CD34(+) human hematopoietic stem/progenitor cells cultured on either OP9 feeder cells ((a), (c)) or C3H10T1/2 feeder cells ((b), (d)) for 7 days ((a), (b)) or 22 days ((c), (d)).
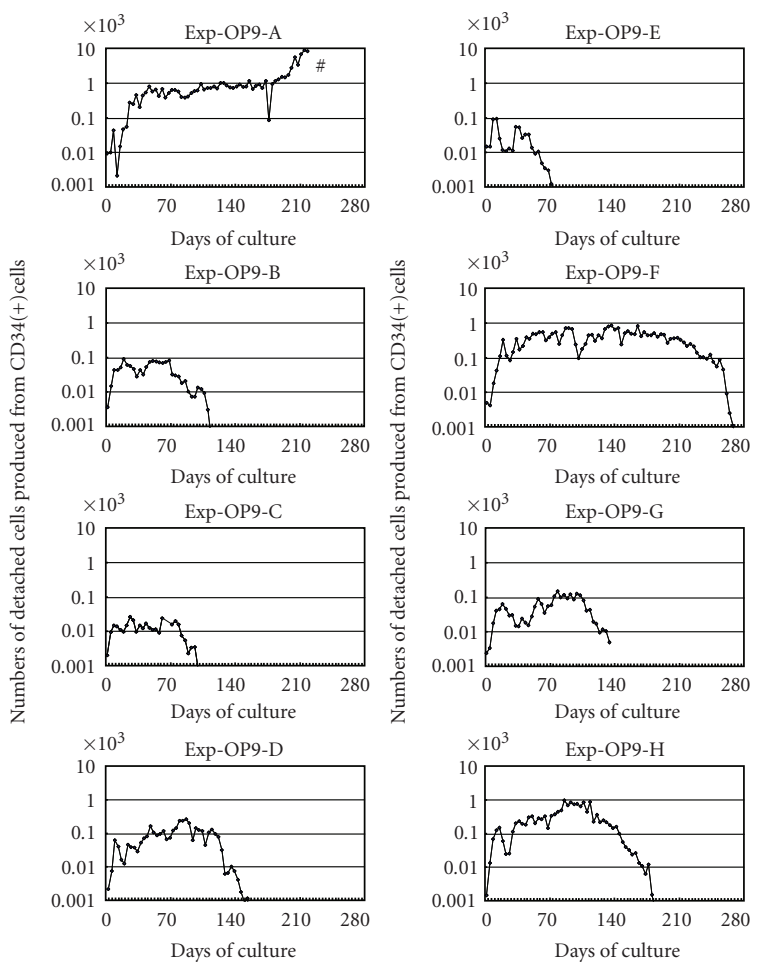

(a)
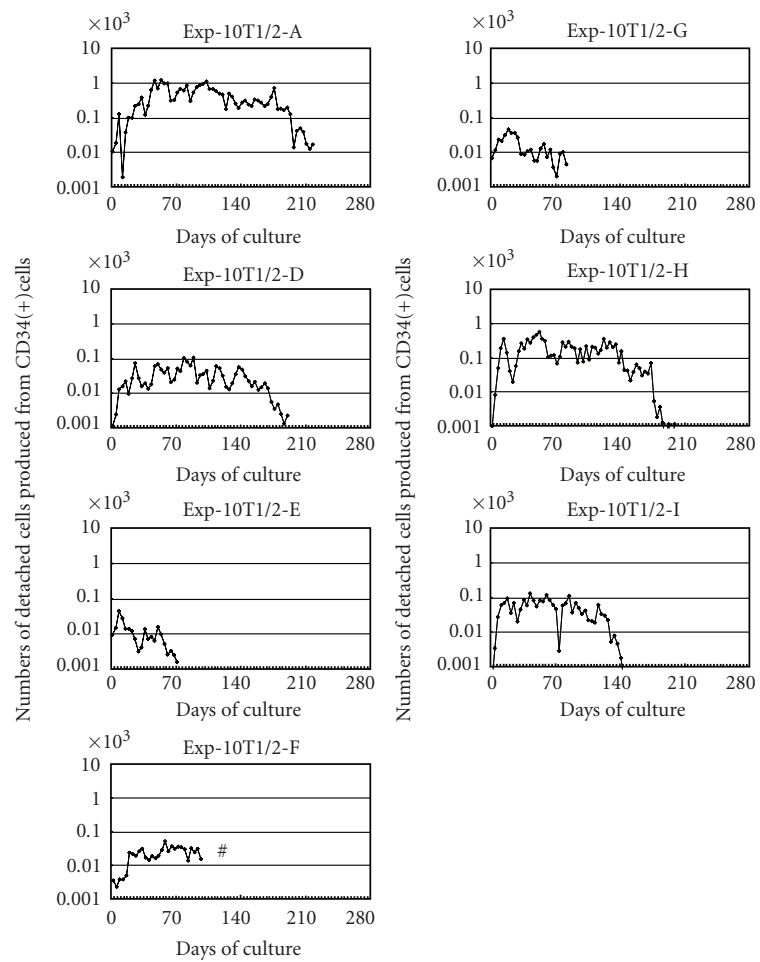

(b)

Figure 2: Production of hematopoietic cells in long-term cultures of human hematopoietic stem cells. (a) Eight independent experiments were performed using eight different umbilical cord blood samples and OP9 cells as feeder cells. (b) Seven independent experiments were performed using seven different umbilical cord blood samples and C3H10T1/2 cells as feeder cells. ((a), (b)) The number of detached cells in the overlying medium was counted at each medium change (approximately half weekly). The data are shown as the mean number of detached cells produced from a single CD34(+) cell, that is, the total number of detached cells divided by the number of CD34 $(+)$ cells used to initiate the culture. Exp: experiment. A to I after Exp-OP9 and Exp-10T1/2 indicate 9 different umbilical cord blood samples derived from 9 different neonates. \#: Cultures Exp-OP9-A and Exp-10T1/2-F were terminated because of fungal infection.

detail below, flow cytometric analysis and a transplantation assay demonstrated that the detached cells produced in this culture method included both mature and immature hematopoietic cells, such as colony-forming cells and hematopoietic stem cells. We found that production of detached cells eventually ceased in all experiments except for Exp-OP9-A. Unfortunately, we were forced to halt ExpOP9-A because of fungal infection although the cells in this culture proliferated efficiently and robustly before fungal contamination (Figure 2).
The numbers of detached cells varied among the experiments (Figure 2) and, notably, showed no correlation with the initial number of $\mathrm{CD} 34^{+}$cells used in each experiment. Thus, $5 \times 10^{3} \mathrm{CD}_{3} 4^{+}$cells were used in Exp-OP9-A and Exp$10 \mathrm{~T} 1 / 2-\mathrm{A}$ and both cultures produced substantial numbers of detached cells over a prolonged period (Figure 2). In contrast, a larger number of CD34 $4^{+}$cells $\left(2 \times 10^{5}\right)$ was used to initiate the Exp-OP9-D and Exp-10T1/2-D cultures, but they produced considerably fewer detached cells (Figure 2). These results indicate that the rate of production of detached 

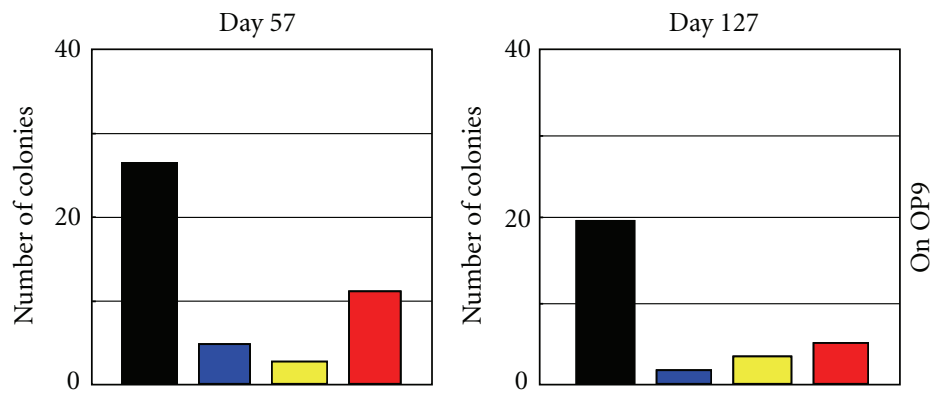
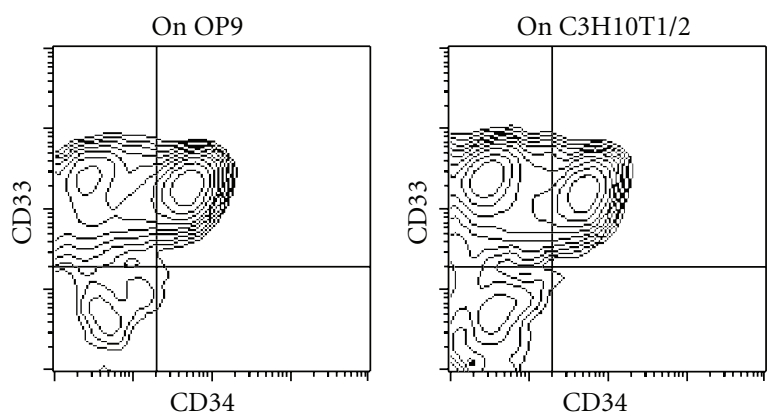

(a)
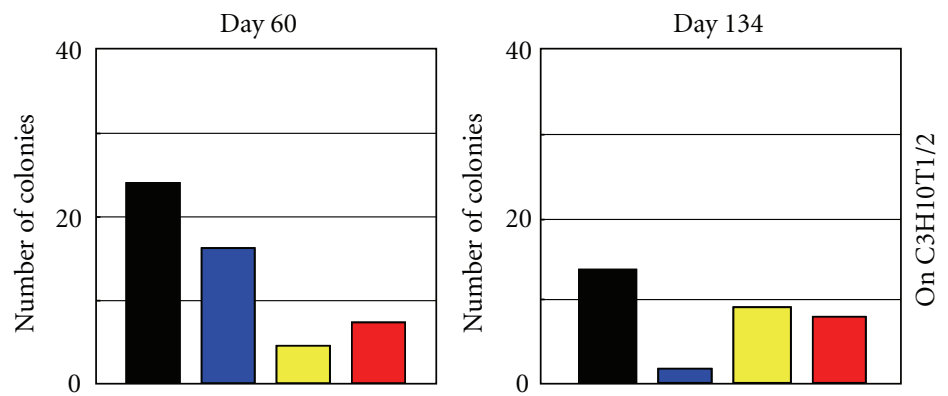

(b)

FIGURE 3: Characterization of cultured cells. (a) Flow cytometric analysis of detached cells produced in cultures on OP9 (Exp-OP9-A) and C3H10T1/2 (Exp-10T1/2-A) feeder cells and collected on Day 218 of culture. The detached cells were stained for CD33, a marker specific for granulocyte/macrophage lineage cells, and CD34, a marker specific for hematopoietic stem/progenitor cells. Flow cytometric analyses of detached cells from other experiments showed similar results. (b) Colony-formation assays. Detached cells produced in culture on OP9 feeder cells (Exp-OP9-A) were collected on Days 57 and 127 of culture. Similarly, detached cells produced in culture on C3H10T1/2 feeder cells (Exp-10T1/2-D) were collected on Days 60 and 134 of culture. The cell samples were used in a standard colony-formation assay. Black bars: colony-forming unit of monocyte/macrophage lineage cells, CFU-M. Blue bars: colony-forming unit of granulocyte lineage cells, CFUG. Yellow bars: colony-forming unit of granulocyte and monocyte/macrophage lineage cells, CFU-GM. Red bars: burst-forming unit of erythroid cells, BFU-E. Similar results were obtained in colony-formation assays using detached cells from other cultures.

cells depended on the quality rather than the number of $\mathrm{CD} 34^{+}$cells used in each experiment. In other words, when the quality of $\mathrm{CD} 34^{+}$cells was high, $5 \times 10^{3} \mathrm{CD} 34^{+}$cells were sufficient to generate efficient in vitro hematopoiesis as shown in Exp-OP9-A and Exp-10T1/2-A (Figure 2).

The majority of detached cells had the morphological characteristics of granulocyte/macrophage lineage cells (Supplementary Figure S1, available at doi 10.1155/2009/936761) although some blast-like cells were also present. Consistent with their morphological phenotype, the majority of the detached cells expressed CD33, a marker of granulocyte/macrophage lineage cells (Figure 3(a)). Of note, $\mathrm{CD} 34^{+} \mathrm{CD} 33^{+}$cells, which are less mature than $\mathrm{CD} 34^{-} \mathrm{CD} 33^{+}$cells, were abundant among the detached cells even at 7 months after initiation of culture (Figure 3(a)).

A colony-formation assay demonstrated that granulocyte, macrophage, and erythrocyte progenitor cells were present among the detached cells (Figure 3(b)). As mentioned above, when the culture medium was changed, detached cells were removed and were not cultured further. Instead, they were either used in experimental analyses or discarded. As is shown in Figure 2, detached cells were continuously produced, and they included abundant colonyforming cells even at Day 127 and Day 134. As one example, the calculated total number of colony-forming cells present in detached cells at Day 127 (upper right, Figure 3(b)) was 13311, which corresponded to a greater than ten-fold increase in the numbers of colony-forming cells compared to the starting material of this culture, that is, 983 colony-forming cells in $5 \times 10^{3} \mathrm{CD}^{2} 4^{+}$cells. Thus, the culture method we describe here could continuously produce abundant colony-forming cells for several months.

Although a mixed colony (a colony derived from very immature hematopoietic cells) was not observed in the colony formation assay (Figure 3(b)), it nevertheless remained possible that hematopoietic stem cells were present at a very low frequency among the detached cells. Therefore, we performed a transplantation assay in which detached cells were injected into an immunodeficient NOD/Shi-scid IL$2 \mathrm{R} \gamma^{\text {null }}$ (NOG) mouse (mentioned hereafter).

\subsection{Hematopoietic Stem Cells Cultured In Vitro for Several} Months Give Rise to Long-Lasting In Vivo Hematopoiesis after Transplantation into Mice. Detached cells were collected from Exp-OP9-A on Day 169 after initiation of culture and transplanted into an NOG mouse $\left(3.9 \times 10^{6}\right.$ cells $)$ (Figure 4(a)). Peripheral blood samples from the mouse were subjected to flow cytometric analysis on Days 56 and 


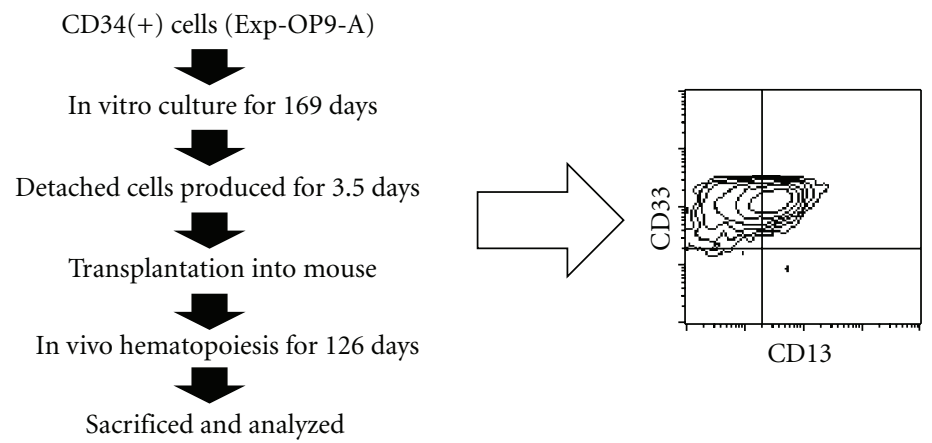

(a)

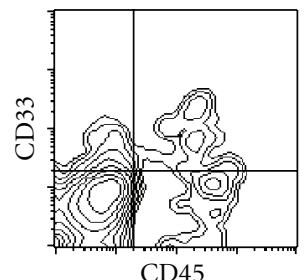

(b)

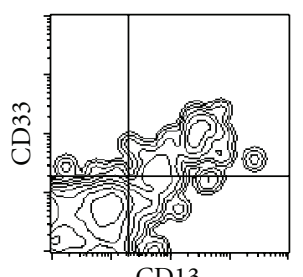

(c)

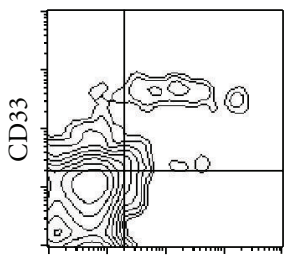

CD13

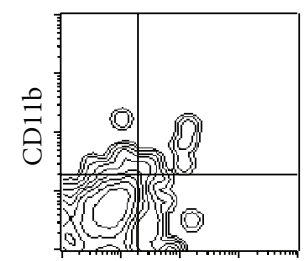

CD14

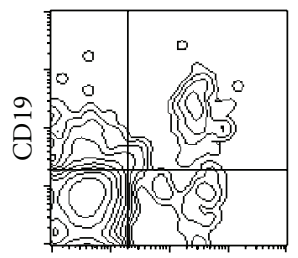

CD45

(d)
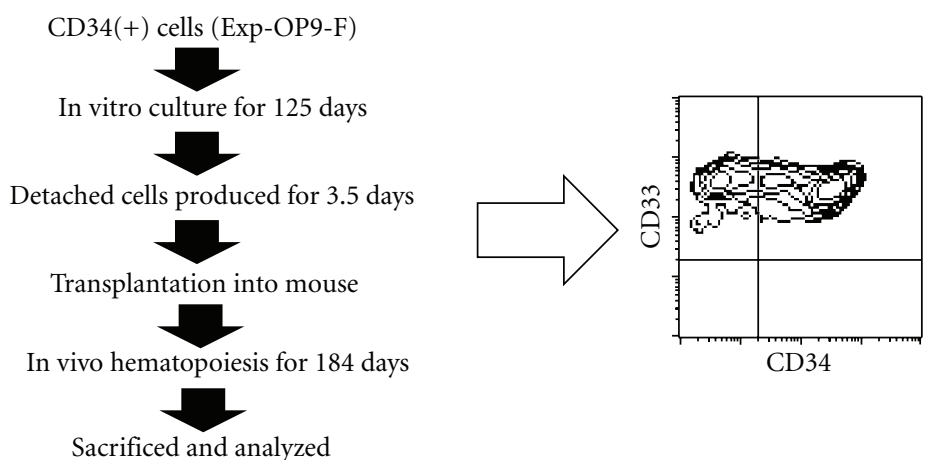

(e)
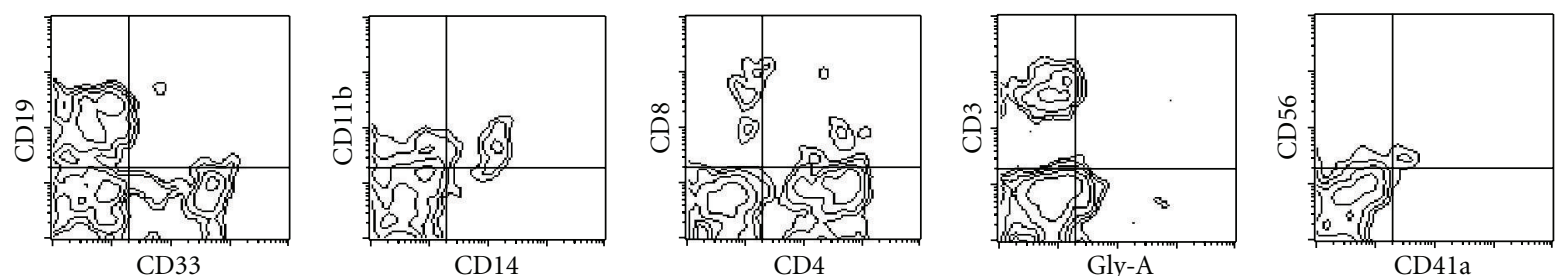

(f)

FIgURE 4: Flow cytometric analysis of hematopoietic cells of the mouse that had been transplanted with human hematopoietic cells produced by in vitro culture. ((a), (e)) Schema of the experimental procedure and flow cytometric analysis of transplanted cells. ((a)(d)) Detached cells $\left(3.9 \times 10^{6}\right.$ cells) produced on OP9 feeder cells (Exp-OP9-A) were collected on Day 169 of culture and transplanted into an immunodeficient NOG mouse. Peripheral blood was collected on Days 56 (b) and 112 (c) after transplantation, and bone marrow cells were collected on Day 126 after transplantation (d). ((e), (f)) Detached cells $\left(2.4 \times 10^{6}\right.$ cells) produced on OP9 feeder cells (ExpOP9-F) were collected on day 125 of culture and transplanted into an immunodeficient NOG mouse. The bone marrow cells were collected on Day 184 after transplantation and were analyzed. ((a)-(f)) The cells were stained using monoclonal antibodies against CD45, a leukocyte common antigen, CD34, a marker specific for hematopoietic stem/progenitor cells, CD33 and CD13, markers of granulocyte and monocyte/macrophage lineage cells, CD11b and CD14, markers of monocyte/macrophage lineage cells, CD19, a marker of B lymphocyte lineage cells, CD3, CD4, and CD8, markers of T lymphocyte lineage cells, Gly-A (Glycophorin A), a marker of erythroid cells, CD56, a marker of large granular lymphocytes and natural killer cells, and CD41a, a marker of megakaryocyte/platelet lineage cells. 
112 after transplantation. Human hematopoietic cells were clearly present in the peripheral bloods (Figures 4(b), 4(c)). The mouse was sacrificed on Day 126 after transplantation, and bone marrow and spleen cells were subjected to flow cytometric analysis. Human hematopoietic cells were present in the bone marrow (Figure $4(\mathrm{~d})$ ) but were present at a very low rate in spleen (data not shown). The estimated rate of chimerism of human $\mathrm{CD} 45^{+}$cells in bone marrow was $2.6 \%$ when compared to the number of mouse CD $45^{+}$ cells. The human hematopoietic cells detected in the bone marrow included cells of the myeloid lineage $\left(\mathrm{CD} 13^{+} \mathrm{CD} 33^{+}\right.$: $9.7 \%$ of the human $\mathrm{CD} 45^{+}$cells), the monocyte/macrophage

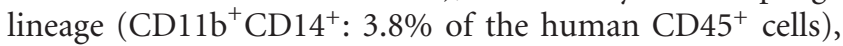
the B cell lineage $\left(\mathrm{CD} 19^{+}: 80.5 \%\right.$ of the human $\mathrm{CD} 45^{+}$cells) (Figure $4(\mathrm{~d})$ ), and other lineages at very low levels (data not shown).

Detached cells were collected from Exp-OP9-F on day 125 after initiation of culture and transplanted into an NOG mouse $\left(2.4 \times 10^{6}\right.$ cells) (Figure 4(e)). CD $45^{+}$ human hematopoietic cells were present in peripheral blood from the mouse one month after transplantation (data not shown). The mouse was sacrificed on Day 184 after transplantation, and the bone marrow cells were subjected to flow cytometric analysis. The rate of chimerism of human $\mathrm{CD}_{4} 5^{+}$cells in the bone marrow was $0.2 \%$ when compared to the number of mouse $\mathrm{CD} 45^{+}$cells. The bone marrow contained human hematopoietic cells, which included cells of the myeloid lineage $\left(\mathrm{CD}_{3} 3^{+}: 23.5 \%\right.$ of the human $\mathrm{CD} 45^{+}$ cells), the monocyte/macrophage lineage $\left(\mathrm{CD}_{11 b^{+}} \mathrm{CD}_{14}{ }^{+}\right.$: $6.7 \%$ of the human $\mathrm{CD} 45^{+}$cells), as well as the $\mathrm{B}\left(\mathrm{CD} 19^{+}\right.$: $53.7 \%$ of the human $\mathrm{CD}^{+} 5^{+}$cells) and $\mathrm{T}$ cell lineages $\left(\mathrm{CD}^{+}, \mathrm{CD}^{+}, \mathrm{CD}^{+}\right.$, and $\mathrm{CD} 4^{+} \mathrm{CD}^{+}: 10.7 \%$ of the human $\mathrm{CD} 5^{+}$cells) (Figure 4(f)). Erythroid (Glycophorin $\mathrm{A}^{+}$) and megakaryocyte $\left(\mathrm{CD} 41 \mathrm{a}^{+}\right)$lineage cells were present at very low rates (Figure $4(\mathrm{f})$ ).

In the transplantation assay described above, human hematopoietic cells of various lineages were present in mice up to six months after cell transplantation. In general, it is impossible that in vivo hematopoiesis derived from transplanted cells is maintained for several months solely by committed progenitor cells. Therefore, the transplanted cells appeared to include hematopoietic stem cells, that is, even after in vitro culture for several months, hematopoietic stem cells were still present in cultures Exp-OP9-A and Exp-OP9F. In light of the numbers of cells produced in culture and of the duration of this production, the hematopoietic stem cells also appeared to have survived for several months in ExpOP9-H, Exp-10T1/2-A, and Exp-10T1/2-H.

The in vitro expansion of human hematopoietic stem cells is known to be very difficult [3]. In agreement with this, a mixed colony (a colony derived from very immature hematopoietic cells) was not observed in the colony formation assay in this study (Figure 3(b)), indicating that hematopoietic stem cells did not expand to any great extent in our culture method. However, since it was highly unlikely that in vitro hematopoiesis could be maintained for several months solely by committed progenitor cells present in the starting materials, the long-lasting in vitro hematopoiesis was likely maintained by hematopoietic stem cells.
On the basis of a previous estimation of the numbers of hematopoietic stem cells capable of repopulating in NOD/SCID mice [19], the starting materials we used in Exp-OP9-A and Exp-OP9-F should have included a very low number of NOD/SCID-repopulating cells. However, the transplantation assay demonstrated that NOD/SCIDrepopulating cells were present among the detached cells that were continuously produced in our culture method (Figure 4), strongly suggesting that our culture method continuously produced small numbers of new NOD/SCIDrepopulating cells throughout the long-term culture period. Hence, the total number of NOD/SCID-repopulating cells that were produced as detached cells throughout the whole long-term in vitro culture was likely greater than the number of such cells that were present in the starting materials.

Taken together, the hematopoietic stem cells capable of repopulating in NOD/SCID mice in our culture system appeared to be maintained by asymmetric cell division, that is, one of the daughter cells retained the characteristics of hematopoietic stem cells and another did not.

Leukemic stem cells might also survive and/or proliferate in our culture method for a prolonged period, enabling basic research or screening for effective anticancer drugs to be performed on these cultured cells. In addition, basic research on specific diseases, such as aplastic anemia or paroxysmal nocturnal hemoglobinuria, might benefit from a long-term culture system for hematopoietic stem cells derived from patients.

\section{Conclusions}

To the best of our knowledge, this is the first report to show that human hematopoietic stem cells can survive in vitro for several months. Since the duration of in vitro hematopoiesis appeared to depend on the quality of hematopoietic stem cells present in each sample, our culture method may be of value for assessing the quality of hematopoietic stem cells prior to their use in the clinic. In particular, our method could be used for the evaluation of umbilical cord bloods since these samples are routinely used in the clinic following preservation for several months. For example, in this study the quality of hematopoietic stem cells derived from samples A, F, and $\mathrm{H}$ appeared to be higher compared to other samples.

\section{Acknowledgments}

The authors obtained human umbilical cord blood from the Cell Engineering Division of RIKEN BioResource Center, which was supported by the National Bio-Resources Project and the Stem Cell Resource Network (Banks at Miyagi, Tokyo, Kanagawa, Aichi, and Hyogo) of the Ministry of Education, Culture, Sports, Science, and Technology in Japan (MEXT), and from Dr. Isamu Ishiwata (Ishiwata Hospital, Mito, Ibaraki, Japan). This work was supported by grants from MEXT. The authors thank all members in the Cell Engineering Division for help, discussion, and secretarial assistance. 


\section{References}

[1] K. Le Blanc, F. Frassoni, L. Ball, et al., "Mesenchymal stem cells for treatment of steroid-resistant, severe, acute graft-versushost disease: a phase II study," The Lancet, vol. 371, no. 9624, pp. 1579-1586, 2008.

[2] L. I. Zon, "Intrinsic and extrinsic control of haematopoietic stem-cell self-renewal," Nature, vol. 453, no. 7193, pp. 306$313,2008$.

[3] T. Heike and T. Nakahata, "Ex vivo expansion of hematopoietic stem cells by cytokines," Biochimica et Biophysica Acta, vol. 1592, no. 3, pp. 313-321, 2002.

[4] K. Ando, T. Yahata, T. Sato, et al., "Direct evidence for ex vivo expansion of human hematopoietic stem cells," Blood, vol. 107, no. 8, pp. 3371-3377, 2006.

[5] T. M. Dexter, T. D. Allen, and L. G. Lajtha, "Conditions controlling the proliferation of haemopoietic stem cells in vitro," Journal of Cellular Physiology, vol. 91, no. 3, pp. 335344, 1977.

[6] I. D. Lewis, G. Almeida-Porada, J. Du, et al., "Umbilical cord blood cells capable of engrafting in primary, secondary, and tertiary xenogeneic hosts are preserved after ex vivo culture in a noncontact system," Blood, vol. 97, no. 11, pp. 3441-3449, 2001.

[7] C. L. da Silva, R. Gonçalves, K. B. Crapnell, J. M. S. Cabral, E. D. Zanjani, and G. Almeida-Porada, "A human stromal-based serum-free culture system supports the ex vivo expansion/maintenance of bone marrow and cord blood hematopoietic stem/progenitor cells," Experimental Hematology, vol. 33, no. 7, pp. 828-835, 2005.

[8] P. Feugier, N. Li, D.-Y. Jo, et al., "Osteopetrotic mouse stroma with thrombopoietin, c-kit ligand, and flk-2 ligand supports long-term mobilized CD $34^{+}$hematopoiesis in vitro," Stem Cells and Development, vol. 14, no. 5, pp. 505-516, 2005.

[9] T. Yoshikubo, T. Inoue, M. Noguchi, and H. Okabe, "Differentiation and maintenance of mast cells from CD $34^{+}$human cord blood cells," Experimental Hematology, vol. 34, no. 3, pp. 320-329, 2006.

[10] T. Hiroyama, K. Miharada, N. Aoki, et al., "Long-lasting in vitro hematopoiesis derived from primate embryonic stem cells," Experimental Hematology, vol. 34, no. 6, pp. 760-769, 2006.

[11] T. Nakano, H. Kodama, and T. Honjo, "Generation of lymphohematopoietic cells from embryonic stem cells in culture," Science, vol. 265, no. 5175, pp. 1098-1101, 1994.

[12] T. Nakano, H. Kodama, and T. Honjo, "In vitro development of primitive and definitive erythrocytes from different precursors," Science, vol. 272, no. 5262, pp. 722-724, 1996.

[13] M. A. Vodyanik, J. A. Bork, J. A. Thomson, and I. I. Slukvin, "Human embryonic stem cell-derived CD $34^{+}$cells: efficient production in the coculture with OP9 stromal cells and analysis of lymphohematopoietic potential," Blood, vol. 105, no. 2, pp. 617-626, 2005.

[14] T. Hiroyama, K. Miharada, K. Sudo, I. Danjo, N. Aoki, and Y. Nakamura, "Establishment of mouse embryonic stem cell-derived erythroid progenitor cell lines able to produce functional red blood cells," PLoS ONE, vol. 3, no. 2, article e1544, pp. 1-11, 2008.

[15] N. Takayama, H. Nishikii, J. Usui, et al., "Generation of functional platelets from human embryonic stem cells in vitro via ES-sacs, VEGF-promoted structures that concentrate hematopoietic progenitors," Blood, vol. 111, no. 11, pp. 5298 5306, 2008.
[16] G. Hangoc, R. Daub, R. G. Maze, J. H. Falkenburg, H. E. Broxmeyer, and M. A. Harrington, "Regulation of myelopoiesis by murine fibroblastic and adipogenic cell lines," Experimental Hematology, vol. 21, no. 4, pp. 502-507, 1993.

[17] M. Nishikawa, K. Ozawa, A. Tojo, et al., "Changes in hematopoiesis-supporting ability of $\mathrm{C} 3 \mathrm{H} 10 \mathrm{~T} 1 / 2$ mouse embryo fibroblasts during differentiation," Blood, vol. 81, no. 5, pp. 1184-1192, 1993.

[18] T. L. Maekawa, T. A. Takahashi, M. Fujihara, et al., "A novel gene (drad-1) expressed in hematopoiesis-supporting stromal cell lines, ST2, PA6 and A54 preadipocytes: use of mRNA differential display," Stem Cells, vol. 15, no. 5, pp. 334-339, 1997.

[19] T. Ueda, K. Tsuji, H. Yoshino, et al., "Expansion of human NOD/SCID-repopulating cells by stem cell factor, Flk2/Flt3 ligand, thrombopoietin, IL-6, and soluble IL-6 receptor," The Journal of Clinical Investigation, vol. 105, no. 7, pp. 1013-1021, 2000. 


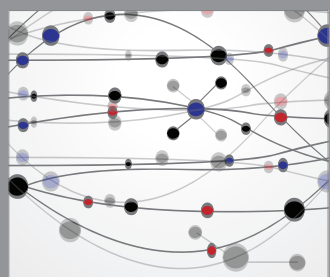

The Scientific World Journal
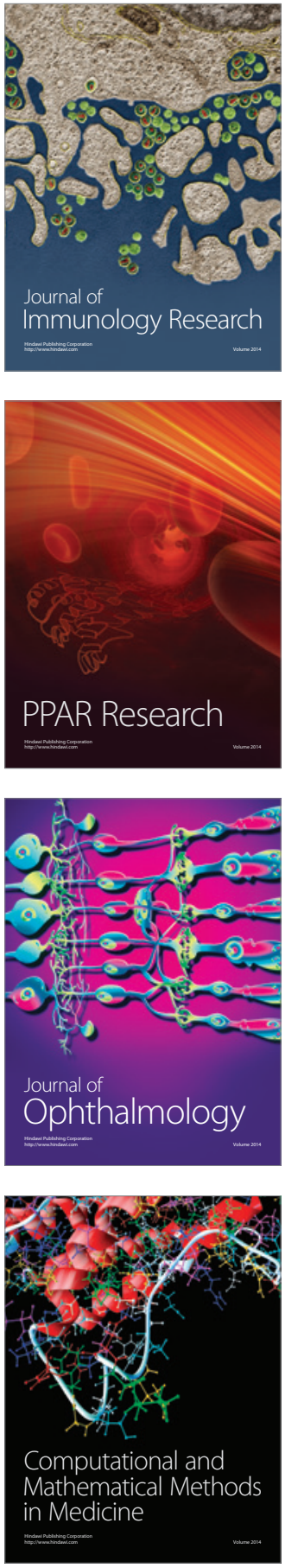

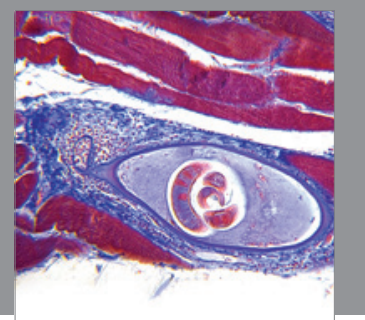

Gastroenterology

Research and Practice
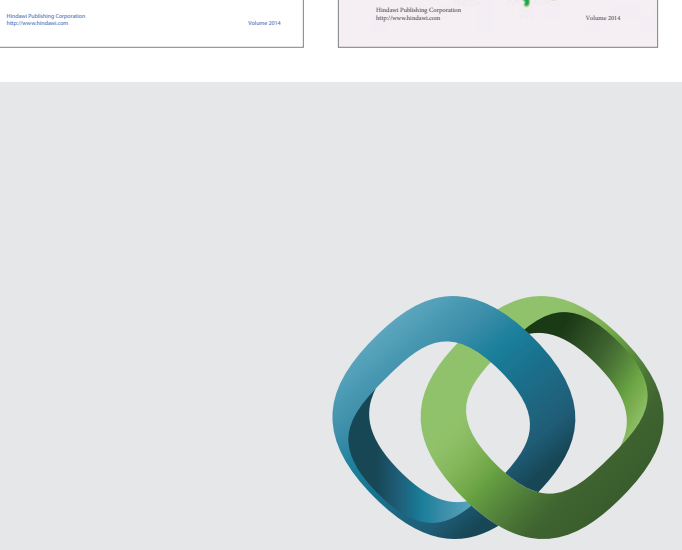

\section{Hindawi}

Submit your manuscripts at

http://www.hindawi.com
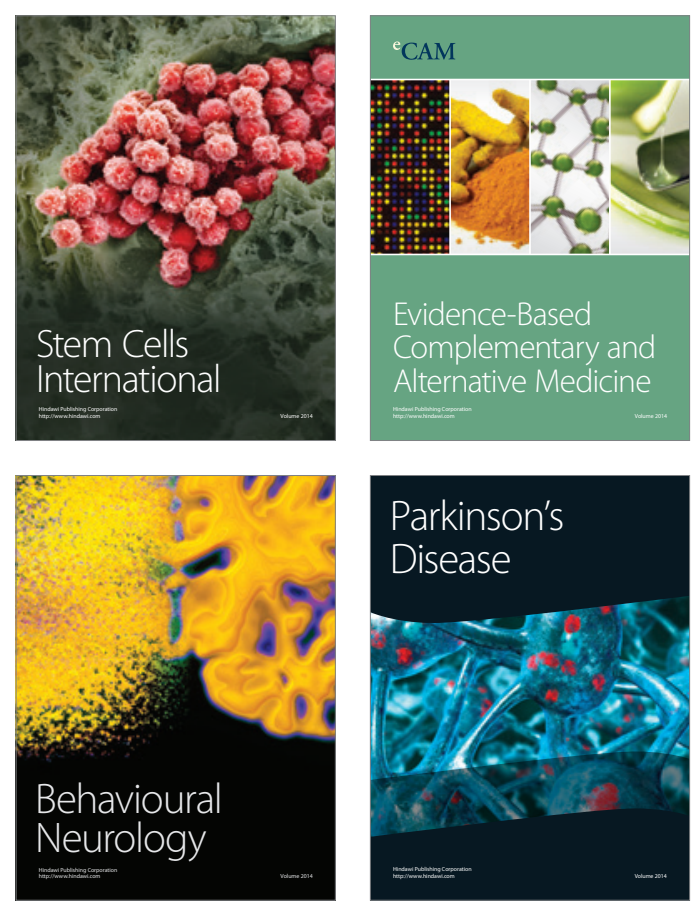

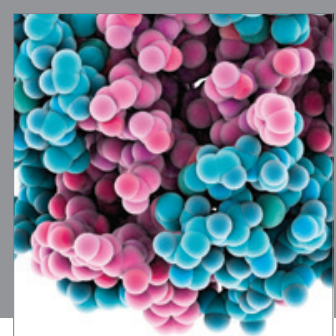

Journal of
Diabetes Research

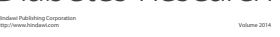

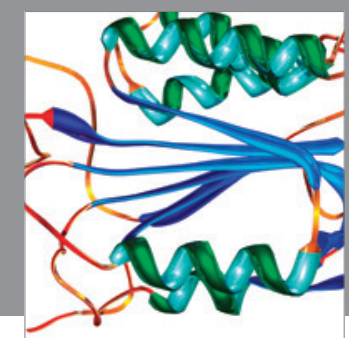

Disease Markers
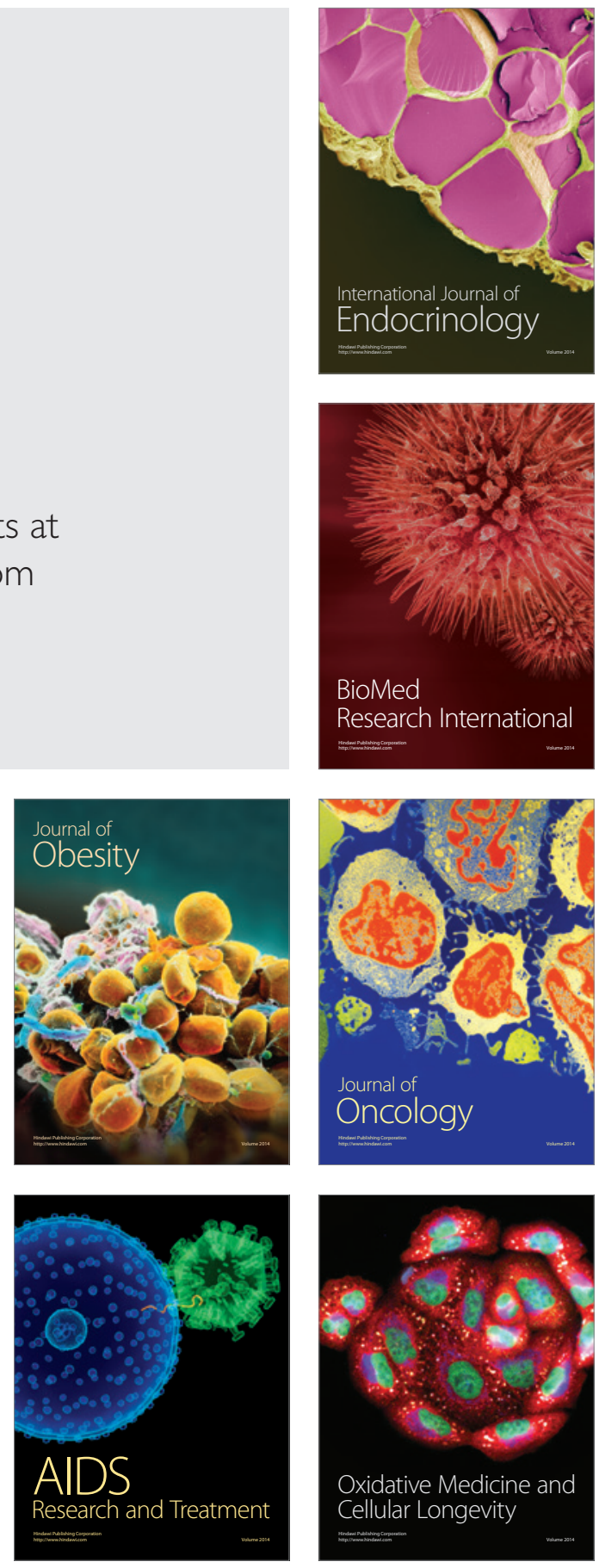